J. Austral. Math. Soc. 25 (Series A) (1978), 41-44

\title{
EXISTENCE OF FINITE GROUPS WITH CLASSICAL COMMUTATOR SUBGROUP
}

\author{
MICHAEL D. MILLER
}

(Received 28 June 1976; revised 3 May 1977)

Communicated by M. F. Newman

\begin{abstract}
Given a group $G$, we may ask whether it is the commutator subgroup of some group $\mathscr{G}$. For example, every abelian group $G$ is the commutator subgroup of a semi-direct product of $G \times G$ by a cyclic group of order 2 . On the other hand, no symmetric group $S_{n}(n>2)$ is the commutator subgroup of any group $\mathscr{G}$. In this paper we examine the classical linear groups over finite fields $K$ of characteristic not equal to 2, and determine which can be commutator subgroups of other groups. In particular, we settle the question for all normal subgroups of the general linear groups $G L_{n}(K)$, the unitary groups $U_{n}(K)(n \neq 4)$, and the orthogonal groups $O_{n}(K)(n \geqslant 7)$.
\end{abstract}

Subject classification (Amer. Math. Soc. (MOS) 1970): 20F35, 20 G40.

\section{Preliminaries}

If $x$ and $y$ are elements of a group $G$, the commutator of $x$ and $y$, written $[x, y]$, is the element $x^{-1} y^{-1} x y$. The commutator subgroup of $G$ is denoted by $G^{\prime}$. We call $G$ a $C$-group if it is the commutator subgroup of some group $\mathscr{G}$. We denote by $o(x)$ the order of $x$, by $x^{*}$ the inner automorphism of $G$ induced by $x$, and by $\langle x\rangle$ the subgroup generated by $x$.

We now give three theorems which are needed later.

THEOREM 1. Let $H$ be a characteristic subgroup of $G, x \in G$. Suppose that there is no element $\varphi \in(\text { Aut } H)^{\prime}$ such that $\left.x^{*}\right|_{H}=\varphi$. Then $G$ is not a C-group.

ProOF. Suppose $\mathscr{G}^{\prime}=G$. As $H$ is characteristic in $G$, and $G$ is characteristic in $\mathscr{G}$, $H$ is characteristic in $\mathscr{G}$. Now $x$ is a product of commutators in $\mathscr{G}$, each of which acts on $H$ (via conjugation) as an element of (Aut $H)^{\prime}$. Hence $\left.x^{*}\right|_{H}=\varphi$ for some $\varphi \in(\text { Aut } H)^{\prime}$, and the result follows.

TheOREM 2. Suppose $\varphi \in$ Aut $G$ has order s. Extend $G$ by the cyclic group $\langle\varphi\rangle$ of order $s$ to obtain a group $G=\langle G, \varphi\rangle$ with relations

$$
\text { those of } G, \varphi^{8}=1, \varphi^{-1} g \varphi=g^{\varphi} \quad(g \in G) .
$$

Then $\bar{G}^{\prime}=\left\langle G^{\prime}, g^{\varphi-1} \mid g \in G\right\rangle$. 
The proof is straightforward and is omitted. Clearly $G^{\prime} \subseteq G$, and if equality holds, we have constructed a group of which $G$ is the commutator. If $x \in G$, we define the $x$-order of $\varphi$, denoted by $o(\varphi, x)$, to be the order of the element $\varphi x$ in $\bar{G}$. It is easy to see that $o(\varphi, x)$ is a multiple of $s$.

THEOREM 3. Let $G$ be a group, $x \in G, \varphi, \psi \in$ Aut $G$ with $[\varphi, \psi]=x^{*}$. Then there exists a group $\mathscr{G}$ with $\mathscr{G}^{\prime} \subseteq G \subseteq \mathscr{G}$ and $x \in \mathscr{G}^{\prime}$.

Proof. We construct $\mathscr{G}$ by consecutive cyclic extensions of $G$. Suppose that $o(\varphi)=s, o(\psi)=t$, and let $o(\varphi, x)=n$. Extend $G$ by the cyclic group $\langle\bar{\varphi}\rangle$ of order $n$ to obtain a group $\bar{G}$ with relations

$$
\text { those of } G, \quad \bar{\varphi}^{n}=1, \quad \bar{\varphi}^{-1} g \tilde{\varphi}=g^{\varphi} \quad(g \in G) .
$$

We now extend $\psi$ to the generators of $\bar{G}$ by defining

$$
\psi: \begin{aligned}
& g \rightarrow g^{\psi} \quad(g \in G), \\
& \bar{\varphi} \rightarrow \bar{\varphi} x .
\end{aligned}
$$

Using the fact that $[\varphi, \psi]=x^{*}$, it is easily checked that this indeed defines an automorphism of $\bar{G}$. We can now extend $G$ by the cyclic group $\langle\bar{\psi}\rangle$ of order $\bar{t}$, where $\bar{t}$ is the order of $\psi$ in Aut $\bar{G}$. We obtain a group $\mathscr{G}$ with relations

$$
\text { those of } G, \quad \begin{aligned}
\bar{\varphi}^{n} & =1, & \bar{\varphi}^{-1} g \bar{\varphi} & =g^{\varphi}, \quad \bar{\psi}^{\bar{l}}=1, \\
\bar{\psi}^{-1} g \bar{\psi} & =g^{\psi}, & \bar{\psi}^{-1} \bar{\varphi} \bar{\psi} & =\bar{\varphi} x .
\end{aligned}
$$

A simple calculation shows that

$$
\mathscr{G}^{\prime}=\left\langle G^{\prime}, x, g^{\varphi-1}, g^{\psi-1} \mid g \in G\right\rangle .
$$

Hence $\mathscr{G}$ has the desired properties.

\section{General linear groups}

Let $G L=G L_{n}(K)$ be the group of non-singular $n \times n$ matrices $(n>1)$ over the finite field $K=\mathbf{F}_{q}$ of $q=p^{k}$ elements $(p>2)$, and let

$$
S L=S L_{n}(K)=\{X \in G L \mid \operatorname{det} X=1\} .
$$

It is known (Dieudonné, 1951) that Aut $S L$ is generated by automorphisms of the following types:

(i) $A \rightarrow X^{-1} A X$, where $X \in G L$.

(ii) $A \rightarrow A^{\sigma}$, where $\sigma \in$ Aut $K$.

(iii) $A \rightarrow\left(A^{-1}\right)^{l}$.

We denote automorphisms of these three types by $\varphi, \chi, \psi$ respectively.

We wish to determine (Aut $S L)^{\prime}$. A simple calculation shows that (Aut $\left.S L\right)^{\prime}$ is generated by the elements $\left[w_{1}, w_{2}\right]$, where $w_{1}, w_{2}$ run through the three types $\varphi, \chi, \psi$. As $G L^{\prime}=S L$, any commutator $\left[\varphi_{1}, \varphi_{2}\right]$ is clearly an inner automorphism of $S L$. Since Aut $K$ is abelian, we have $\left[\chi_{1}, \chi_{2}\right]=1$. 
Suppose $\varphi: A \rightarrow X^{-1} A X$ and $\chi: A \rightarrow A^{\sigma}$. Then $[\varphi, \chi]: A \rightarrow X^{-\sigma} X A X^{-1} X^{\sigma}$. Now $\operatorname{det}\left(X^{-1+\sigma}\right)=(\operatorname{det} X)^{p^{r-1}}$, where $\sigma: K \rightarrow K$ is given by $y^{\sigma}=y^{p^{r}}$ for all $y \in K$. As $p^{r}-1$ is even, $\operatorname{det}\left(X^{-1+\sigma}\right)$ is a square in $K$. Thus $[\varphi, \chi]$ is an automorphism of type (i), induced by an element of $G L$ with square determinant.

Now suppose $\varphi: A \rightarrow X^{-1} A X$, and $\psi: A \rightarrow\left(\mathrm{A}^{-1}\right)^{t}$. Then

$$
[\varphi, \psi]: A \rightarrow X^{i} X A X^{-1}\left(X^{-1}\right)^{l} .
$$

Since $\operatorname{det}\left(X^{i} X\right)$ is a square, $[\varphi, \psi]$ is of type (i), induced by an element of $G L$ with square determinant. Finally, $[\chi, \psi]=1$. We conclude that

(Aut $S L)^{\prime} \subseteq\left\{X^{*} \mid X \in G L\right.$, det $X$ is a square $\}$.

Except for $G L_{2}\left(F_{3}\right)$, every non-central normal subgroup of $G L$ contains $S L$. So let $S$ be such a subgroup, $S L \subseteq S \subseteq G L$. Then $S^{\prime}=S L$ and so $S L$ is characteristic in $S$. Furthermore, $C_{S}(S L)=Z(S)$ and so by Theorem 1, a necessary condition for $S$ to be a $C$-group is that $S / Z(S) \subseteq(\text { Aut } S L)^{\prime}$.

Let $\alpha$ be a generator of $K^{*}$, and let $[G L: S]=r$, so that $S=\{X \in G L \mid \operatorname{det} X$ is an $r$ th power $\}$. If $Q=\operatorname{diag}(\alpha, 1,1, \ldots, 1)$, then $S=\left\langle S L, Q^{r}\right\rangle$. Assume $\mathscr{G}^{\prime}=S$. Since $Q^{r} \in \mathscr{G}^{\prime}$, the above analysis implies that $\left.\left(Q^{r}\right)^{*}\right|_{S L}=\left.A^{*}\right|_{S L}$, where $A \in G L$, and $\operatorname{det} A$ is a square. As $C_{G L}(S L)$ consists of the scalar matrices, there is a $\lambda \in K$ such that $\operatorname{det}\left(\lambda Q^{r}\right)=\alpha^{r} \lambda^{n}$ is a square. If $n$ is even and $r$ is odd, we clearly have a contradiction. Hence in such cases, $S$ is not a $C$-group.

Suppose now that $r$ is even. Let $B=\operatorname{diag}\left(\alpha^{r / 2}, 1,1, \ldots, 1\right)$, and consider the following two automorphisms of $S$ :

$$
\begin{aligned}
& \varphi: A \rightarrow B^{-1} A B, \\
& \psi: A \rightarrow\left(A^{-1}\right)^{l} .
\end{aligned}
$$

We find that $\left[\varphi^{-1}, \psi^{-1}\right]=\left(B B^{\imath}\right)^{*}$. But $B B^{l}=Q^{r}$. Define $\mathscr{G}=\langle S, \varphi, \psi\rangle$, with relations as defined in Theorem 3. Then $\mathscr{G}^{\prime}=S$ and so $S$ is a $C$-group.

Finally, assume that both $n$ and $r$ are odd. Let $C=\operatorname{diag}\left(\alpha^{(n+r) / 2}, 1,1, \ldots, 1\right)$, and consider the following two automorphisms of $S$ :

$$
\begin{aligned}
& \varphi: A \rightarrow C^{-1} A C, \\
& \psi: A \rightarrow\left(A^{-1}\right)^{t} .
\end{aligned}
$$

We have $\left[\varphi^{-1}, \psi^{-1}\right]=\left(C C^{t}\right)^{*}=\left(C C^{t} Z\right)^{*}$, where $Z=\alpha^{-1} I$. But

$$
C C^{t} Z=\operatorname{diag}\left(\alpha^{n+r-1}, \alpha^{-1}, \alpha^{-1}, \ldots, \alpha^{-1}\right)
$$

has determinant $\alpha^{r}$ and so is in $S$. In fact, $S=\left\langle S L, C C^{l} Z\right\rangle$ since $\alpha$ is a generator of $K^{*}$. If we define $\mathscr{G}=\langle S, \varphi, \psi\rangle$ with relations as in Theorem 3 , then $\mathscr{G}^{\prime}=S$.

We may summarize the above results as follows:

THEOREM 4. Let $S$ be a subgroup of $G L_{n}(K)$, char $K \neq 2$, with $S L_{n}(K) \subseteq S \subseteq G L_{n}(K)$, and $\left[G L_{n}(K): S\right]=r$. Then $S$ is a $C$-group except when $n$ is even and $r$ is odd.

It is easily checked that every proper normal subgroup of $G L_{2}\left(\mathbf{F}_{3}\right)$ is a $C$-group and so the theorem is true for any normal subgroup $S$ of $G L_{n}(K)$. 


\section{Orthogonal and unitary groups}

Let $K$ be the finite field of $p^{h}$ elements $(p>2)$, and suppose that $f$ is a nondegenerate symmetric bilinear form on a $K$-vector space $V$ with index $\nu(f) \geqslant 1$. Denote by $O_{n}(K, f)$ the corresponding orthogonal group. If $\left\{e_{i}\right\}, i=1,2, \ldots, n$, is an orthogonal basis for $V$, and $R$ is the (diagonal) matrix of $f$ with respect to this basis, then $O_{n}(K, f)$ is realized as the set of all $A \in G L_{n}(K)$ with $A R A^{t}=R$. Let $\Omega_{n}(K, f)$ denote the commutator subgroup $O_{n}(K, f)^{\prime}$ and set

$$
O_{n}^{+}(K, f)=\left\{A \in O_{n}(K, f), \operatorname{det} A=1\right\} .
$$

Suppose now that $h$ is even, so that $K$ has a unique non-trivial involution $\sigma$, where $y^{\sigma}=y^{p^{h / 2}}$ for all $y \in K$. Let $g$ be a reflexive $\sigma$-linear form on $V$, and denote by $U_{n}(K, g)$ the corresponding unitary group. With respect to a suitable basis, $U_{n}(K, g)$ is realized as the set of all $A \in G L_{n}(K)$ with $A \tilde{A}=I$, where $\tilde{A}=\left(A^{\sigma}\right)^{t}$. Finally, set $U_{n}^{+}(K, g)=\left\{A \in U_{n}(K, g) \mid \operatorname{det} A=1\right\}$.

Using arguments similar to those used in the general linear case, we obtain the following:

THEOREM 5. Suppose $n \geqslant 7$. If $n$ is odd, the only non-central normal subgroups of $O_{n}(K, f)$ which are $C$-groups are $\Omega_{n}(K, f)$ and $\left\langle\Omega_{n}(K, f),-I\right\rangle$. If $n$ is even, the only such $C$-groups are $\Omega_{n}(K, f)$ and $O_{n}^{+}(K, f)$.

THEOREM 6. Let $S$ be a subgroup of $U_{n}(K, g)$, char $K \neq 2, n \neq 4$, with

$$
U_{n}^{+}(K, g) \subseteq S \subseteq U_{n}(K, g)
$$

and $\left[U_{n}(K, g): S\right]=r$. Then $S$ is $a C$-group except when $n$ is even and $r$ is odd.

\section{References}

E. Artin (1957), Geometric Algebra, Interscience Tracts in Pure and Applied Mathematics, 3 (Interscience, New York, London).

J. Dieudonné (1951), On the Automorphisms of the Classical Groups, Memoirs Amer. Math. Soc. 2 (Amer. Math. Soc., New York).

J. Dieudonné (1958), Sur les Groupes Classiques, Actualités scientifiques et industrielles, 1040 (Publications de l'Institut de Mathématique de l'Université de Strasbourg, VI. Hermann, Paris).

J. Dieudonné (1971), La Géométrie des Groupes Classiques, 3rd ed., Ergebnisse der Mathematik und ihrer Grenzgebiete, 5 (Springer-Verlag, Berlin, Heidelberg, New York).

R. Lipschitz (1959), "Correspondence", Ann. of Math. (2) 69, 247-251.

O. T. O'Meara (1968), "The automorphisms of the orthogonal groups $\Omega\left(V_{n}\right)$ over fields", Amer. J. Math. 90, 1260-1306.

F. D. Veldkamp (1965), Classical Groups (Yale Notes, New Haven, Conn.).

Department of Mathematics

University of California, Los Angeles

California 90024, USA 